\title{
Time-course of infection and responses in a coughing rat model of pertussis
}

\author{
ELIZABETH HALL, R. PARTON* and A. C. WARDLAW \\ Division of Infection and Immunity, Institute of Biomedical and Life Sciences, Joseph Black Building, \\ University of Glasgow, Glasgow G12 800
}

\begin{abstract}
Adult female Sprague-Dawley rats were challenged intrabronchially with Bordetella pertussis strain 18-323 embedded in fine agarose beads and the time-course of infection and other events was determined. There was a steady decline in the numbers of $B$. pertussis recovered from the rat lungs, with clearance of the infection in most animals by day 12. Leucocytosis, lung inflammation and an increase in total serum IgE in the rats as a result of the challenge were highest around day 10 , which was coincident with the highest incidence of coughing in such animals. IgG and IgA antibodies to the $B$. pertussis antigens pertussis toxin and filamentous haemagglutinin were not detected until after this period. The coughing rat model of pertussis resembles the human disease in the relationship between the time course of infection and cough production.
\end{abstract}

\section{Introduction}

Pertussis is a disease only of man and, although various species of laboratory animals may be infected by the respiratory route with Bordetella pertussis, only rats $[1,2]$ and some primates [3] have been reported to develop a paroxysmal coughing syndrome. In recent years, these observations have led to the development of a coughing rat model for investigating pertussis pathogenesis and immunity [4-8]. In adult SpragueDawley rats, paroxysmal coughing started c. 5 days after intrabronchial challenge with $B$. pertussis strain 18-323 contained in minute agarose beads and could be detected for up to 21 days, with the most intense coughing occurring between days 8 and $14[4,5]$. The rats also exhibited marked leucocytosis and retardation of weight gain. Other phase I $B$. pertussis strains also induced coughing whereas, with strains lacking pertussis toxin (PT), much less coughing was heard [6]. When four rat strains were compared, the SpragueDawley animals gave the most coughs [7].

In an early report of cough production in rats after challenge with $B$. pertussis, Woods et al. [2] infected adult male $(200-220 \mathrm{~g})$ Sprague-Dawley rats intrabronchially with $5 \times 10^{5} \mathrm{cfu}$ of $B$. pertussis strain Tohama phase I embedded in agar beads. Bacteria were recovered from the lungs at 3 and 7 days after

Received 23 March 1998; accepted 17 April 1998.

${ }^{*}$ Corresponding author: Dr. R. Parton. challenge and again at day 21 , but not at days 10 and 14. The method of intrabronchial infection employed in the present study differed from that of Woods et al. in several respects, namely: the use of low-gellingtemperature agarose rather than agar to make the beads and avoid mineral oil in the bead-making process [7]; a much higher challenge dose $\left(c .10^{8} \mathrm{cfu}\right)$ of $B$. pertussis strain 18-323 rather than strain Tohama; the use of female Sprague-Dawley rats (125$150 \mathrm{~g}$ ) instead of males (200-220 g); Hypnorm/ Hypnovel as anaesthetic instead of ether; and the use of sound-activated tape recorders to monitor coughing [3-5]. The main objective in these studies was to optimise the conditions whereby coughing was produced and to determine serological responses. Therefore, the rats were not killed until several weeks after challenge. In this study, the time course of infection has been the main focus of interest, in conjunction with other events in the infected rats.

\section{Materials and methods}

\section{Animals and $B$. pertussis challenge}

A total of 75 female Sprague-Dawley rats of high health status was purchased from Harlan Olac Ltd (Shaws Farm, Blackthorn, Bicester) and used in six experiments over 12 months, the results of which are presented collectively. At six weeks of age (125$150 \mathrm{~g}$ ), 64 of the rats were infected intrabronchially with $B$. pertussis 18-323 in agarose beads as described previously [7] and killed at intervals during the first 
month after challenge. The challenge dose range was $(1.25-2.0) \times 10^{8} \mathrm{cfu} /$ rat. Samples were taken from untreated controls on days $10(n=5)$ and $28(n=6)$.

\section{Post-infection procedures}

The numbers of test rats sampled on days $1,3,7,10$, 12,16 and 28 were $16,17,8,14,3,3$ and 3 , respectively. Rats were anaesthetised with halothane and bled by cardiac puncture. Tracheo-bronchial washings (TBW) were taken by cannulation of the trachea and washing out the lungs with $5 \mathrm{ml}$ of sterile phosphate-buffered saline (PBS), of which c. $3.5 \mathrm{ml}$ was recovered. The lungs were then removed aseptically, weighed and homogenised in $10 \mathrm{ml}$ of sterile PBS with a Silverson homogeniser. Samples $(0.1 \mathrm{ml})$ of 10 -fold dilutions of TBW or lung homogenate were plated out on Bordet-Gengou medium (Difco) containing horse blood $20 \% \mathrm{w} / \mathrm{v}$ and incubated for 5 days at $37^{\circ} \mathrm{C}$. B. pertussis colonies were counted and expressed as total cfu/rat, after taking account of the lung weight, volume of TBW recovered and the dilution of the sample for viable counting. The limit of detection for the lung count was $c .130 \mathrm{cfu}\left(\log _{10}=2.1\right) / \mathrm{rat}$, assuming the average lung weight was $3 \mathrm{~g}$, and for TBW was c. $35 \mathrm{cfu}\left(\log _{10}=1.5\right)$, assuming the average volume of TBW recovered was $3.5 \mathrm{ml}$. The white blood cell (WBC) count was measured from blood samples taken when the rats were killed, and coughing was recorded with the use of sound-activated tape recorders and tapes of 45-min duration, as described previously [5]. Recordings were made during the nights of days 8 and 9 after challenge and the number of paroxysms/rat was calculated.

\section{Serology}

Serological measurements of total IgE, and of IgG and IgA specific for two major $B$. pertussis antigens, filamentous haemagglutinin (FHA) and PT, in serum and TBW were made by ELISA as described previously $[5,7]$.

\section{Results and discussion}

The numbers of $B$. pertussis recovered from the rat lungs at different times after challenge are shown in Fig. 1a. On day 1, the mean count in the lung homogenate was $c$. $10^{8} \mathrm{cfu}$, similar to the initial challenge dose of $(1.25-2.0) \times 10^{8} \mathrm{cfu}$. Thereafter, the lung count declined steadily to day 10 . On day 10 , 9 of 14 samples were sterile, although the $\log _{10}$ of the mean of the others was 3.0. No bacteria were detected on days 12,16 and 28 . For graphical purposes, these samples were assigned a notional $\log _{10}$ value of 2.0 , (just below the detection limit of 2.1) and indicated by downward arrows. Fewer bacteria were recovered from TBW samples than from lung homogenates but, in general, a parallel decline in numbers was evident. On

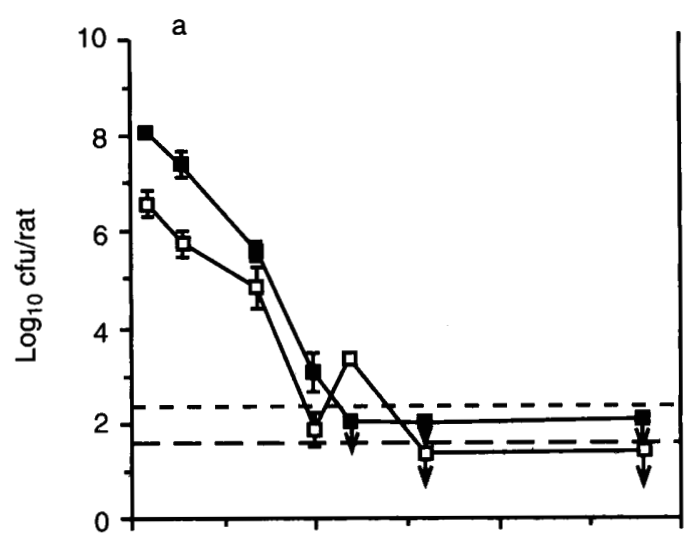

b
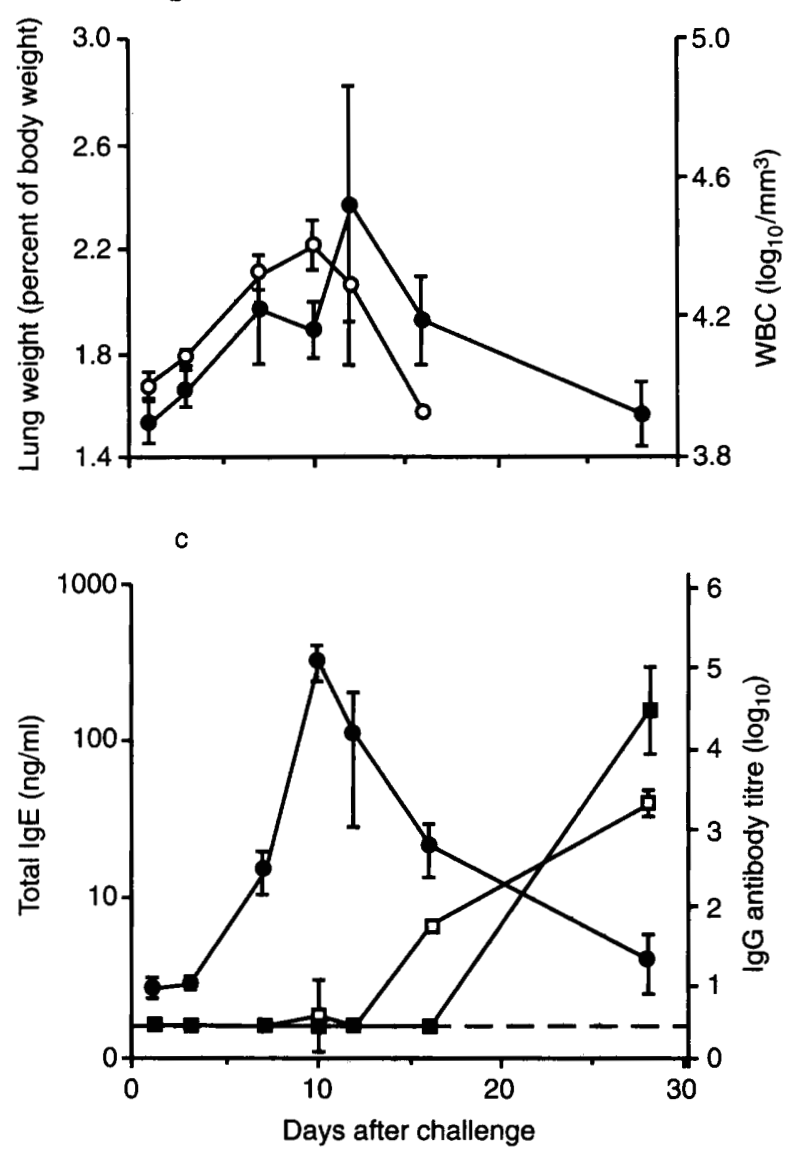

Fig. 1. Time course of events after intrabronchial challenge of rats with $B$. pertussis 18-323. Data are the means (and SEM) from 16, 17, 8, 14, 3, 3 and 3 rats on days $1,3,7,10,12,16$, and 28 , respectively. (a) Recovery of $B$. pertussis $18-323\left(\log _{10} \mathrm{cfu} /\right.$ rat) from lung homogenates (- - ) and tracheo-bronchial washings (TBW) ( $-\square-)$ of infected rats. The limits of detection are indicated for lung homogenates (-) and for TBW $(--)$. (b) Lung weights as a percentage of body weight (--) and white blood cell counts $\left(\log _{10} \mathrm{WBC} / \mathrm{mm}^{3}\right)\left(-\mathrm{O}^{-}\right)$. (c) Serum levels of total IgE (-O) and $\log _{10}$ IgG antibody titres to FHA ( $-\square-$ ) and PT $(-\square-)$. The dashed line at the $\log _{10}$ value of 0.47 is the limit of detection of IgG antibodies by ELISA

day 10 , the same 9 of 14 rats as above yielded no viable $B$. pertussis. The three TBWs sampled on day 12 had a $\log _{10}$ mean count of 3.3 and were followed on days 16 and 28 by samples with no detectable bacteria 
(assigned a $\log _{10}$ value of 1.3 ). As the numbers of samples $(\mathrm{n}=3)$ on days 12,16 and 28 were small, this apparent reappearance of organisms at day 12 could be attributed to variability between rats or experiments. Lung and TBW samples from untreated controls on days 10 and 28 did not contain viable $B$. pertussis.

Lung weights were used as an indication of inflammatory changes after challenge and mean lung weights as a percentage of body weight are shown in Fig. $1 \mathrm{~b}$. The lungs increased in weight after challenge, from 1.54 (SEM 0.08$) \%$ on day 1 , to a peak of 2.38 (SEM 0.45 ) $\%$ between days 8 and 16 , and then declined to 1.56 (SEM 0.12$) \%$ by day 28 . The day 1 and day 28 values were higher than the untreated control values which were 1.19 (SEM 0.15)\% and 1.13 (SEM 0.06$) \%$ on days 10 and 28 , respectively.

As observed in previous studies $[4,5]$, the leucocyte counts in the challenged rats also showed a distinct peak, at c. $25000 \mathrm{WBC} / \mathrm{mm}^{3}\left(\log _{10}=4.4\right)$ around day 10 after infection and then returned to a nearnormal level by day 16 (Fig. 1b). The mean WBC level in untreated, control rats on both days 10 and 28 was c. $8000 \mathrm{WBC} / \mathrm{mm}^{3}\left(\log _{10}=3.9\right)$.

In previous studies with $B$. pertussis-challenged rats, paroxysmal coughing started $c .5$ days after challenge, with most coughing occurring between days 8 and 14 $[4,5]$. In the present study, the occurrence of coughing was confirmed by monitoring the rats on days 8 and 9 , with 6.5 and 7.2 paroxysms/rat recorded, respectively, similar to levels in previous studies [6-8]. Coughing was not detected in untreated controls. Also, as in previous studies $[4,7]$, the challenged animals were $10-20 \%$ lighter in body weight between day 1 and day 10, than untreated controls, but there was little difference between test and control animals by day 28 (data not shown).

The time course of serum antibody production in $B$. pertussis-challenged rats is shown in Fig. 1c. A 100fold increase in total serum $\operatorname{IgE}$ to $c .320 \mathrm{ng} / \mathrm{ml}$ was seen between day 1 and day 10 after challenge; thereafter it declined. Levels of total serum $\operatorname{IgE}$ in untreated control animals on days 10 and 28 were $1-5 \mathrm{ng} / \mathrm{ml}$. This increase in IgE in infected rats occurred before the development of serum IgG specific to the $B$. pertussis FHA and PT antigens. These latter responses increased from day 16 to develop into strong titres by day 28 . Serum IgA antibodies to FHA and PT were not found at day 28, but were detected at day 42 (not shown).

In the TBW of $B$. pertussis-challenged rats, total IgE was present in very much lower concentrations than in serum and near to the limit of detection $(0.05 \mathrm{ng} / \mathrm{ml})$ at all sampling times (not shown). The highest levels were detected in TBW samples at day 12, with a mean value of $0.28(\mathrm{SEM} \mathrm{0.12)} \mathrm{ng} / \mathrm{ml}$. IgE was not detectable $(\leqslant 0.05 \mathrm{ng} / \mathrm{ml})$ in TBW from untreated control animals on days 10 and 28. IgG and $\operatorname{IgA}$ antibodies to FHA and PT were readily detectable in TBW on day 28 , with mean $\log _{10}$ IgG titres of 1.9 (SEM 0.17) and 0.9 (SEM 0.39), and mean log IgA titres of 2.4 (SEM 0.14) and 0.6 (SEM 0.21), for FHA and PT respectively.

The late appearance of $\operatorname{IgA}$ in serum and its earlier appearance in lung washings are in agreement with studies on $B$. pertussis-infected mice $[9,10]$. The timing and magnitude of the $\operatorname{IgA}$ response in TBW suggests its derivation from local origins, rather than by transudation from the serum. IgE may also be produced locally in the respiratory tract [11], but there was little evidence for this or for increased IgE in TBW as a result of the elevated serum levels of this immunoglobulin between days 7 and 16. It may be that much of the IgE in the respiratory tract is bound to the abundant mast cells in this site [12] and, therefore, not detectable in lung washings. B. pertussis cells and PT are well known potentiators of IgE production. The marked elevation of serum $\operatorname{IgE}$ in the $B$. pertussis-challenged rats was coincident with the peak of cough induction, but the significance of these observations is unclear. $B$. pertussis antigen-specific IgE antibodies were not detected. Previous work has shown that such antibodies were below the limit of detection and did not contribute to the elevated levels of circulating $\operatorname{IgE}$ in the rats [7]. A rise in total serum $\operatorname{IgE}$ has been reported in some children with pertussis [13] and a low incidence of $B$. pertussis-specific IgE antibodies has been detected [14].

There are notable differences between mice and rats in their patterns of clearance of $B$. pertussis from the respiratory tract. In adult mice, after intranasal or aerosol infection with a sublethal dose of $c \cdot 10^{5} \mathrm{cfu}$, there was up to 100-1000-fold multiplication within 1-2 weeks, before a gradual decline over several weeks $[3,10]$. With adult Sprague-Dawley rats challenged with agar-embedded $B$. pertussis by a technique similar, but not identical, to that used here, Woods et al. [2] were able to isolate the organisms from lung homogenates on days $1-7$ and at day 21 , but not at days 10 and 14 . With an initial challenge dose of $5 \times 10^{5} \mathrm{cfu} / \mathrm{rat}$, there was $c$. 100 -fold multiplication by day 3 and thereafter a rapid decline in numbers.

The differences between the present findings and those of Woods et al. [2] are probably caused by procedural differences. In the present study, there was no apparent early multiplication, but a steady decline in numbers. In many animals $B$. pertussis had disappeared by day 10 . However, as the challenge consisted of $B$. pertussis embedded in agarose and made into a bead suspension by homogenisation, the counts of the lung homogenates and TBW were likely to have been an underestimate of the actual 
numbers of viable bacteria present, because of containment of the bacteria within undisrupted beads. In a previous study, rats challenged with heat-killed $B$. pertussis in agarose beads, or with live organisms in suspension (without agarose) showed no cough induction or leucocytosis [4]. Therefore, this suggests that with the usual bead challenge, some bacterial multiplication and toxin production do occur, to induce coughing, leucocytosis and other responses, and that the actual lung counts are the result of bacterial proliferation, killing or clearance by the host, and the problem of enumeration referred to above.

Younger rats were more susceptible to infection than older ones, and showed more pronounced symptoms in terms of coughing, leucocytosis and, in some cases, death [7]. In experiments similar to those reported above in which 4-week-old female rats were infected with $c .10^{8} \mathrm{cfu}$ of $B$. pertussis, lung and TBW counts increased $c$. 10 -fold between days 1 and 3, before the steep decline to day 10 as seen in 6-week-old animals. However, because the challenge dose of $c .10^{8} \mathrm{cfu}$ was toxic for these younger rats, they were not considered suitable for use in the coughing rat model.

In human pertussis, the organisms can be isolated from nasal swabs most frequently during the early stages of the disease and become increasingly difficult to culture as the paroxysmal stage progresses [15]. In the rat model, the greatest incidence of coughing occurs between days 8 and $14[4,5]$, coincident with the time when the bacterial numbers are in rapid decline or the infection has been cleared from the lungs. Thus, with respect to the relationship between infection and clinical disease, the coughing rat model of pertussis resembles the human condition. In addition, the parallel between serum IgE and cough production in the rat and the finding of elevated IgE levels in some pertussis patients merits further study.
We gratefully acknowledge the skilled technical assistance of Mrs Berit Adam. These studies were funded by research grant K/MRS/50/C2235 from the Scotttish Office Home and Health Department.

\section{References}

1. Hornibrook JW, Ashburn LL. A study of experimental pertussis in the young rat. Public Health Rep 1939; 54: 439-444.

2. Woods DE, Franklin R, Cryz SJ, Ganss M, Peppler M, Ewanowich C. Development of a rat model for respiratory infection with Bordetella pertussis. Infect Immun 1989; 57: $1018-1024$.

3. Sato Y, Sato H. Animal models of pertussis. In: Wardlaw AC, Parton $R$ (eds) Pathogenesis and immunity in pertussis Chichester, Wiley. 1988; 305-325.

4. Wardlaw AC, Hall E, Parton R. Coughing rat model of pertussis. Biologicals 1993; 21: 27-29.

5. Hall E, Parton R, Wardlaw AC. Cough production, leukocytosis and serology of rats infected intrabronchially with Bordetella pertussis. J Med Microbiol 1994; 40: 205-213.

6. Parton R, Hall E, Wardlaw AC. Responses to Bordetella pertussis mutant strains and to vaccination in the coughing rat model of pertussis. J Med Microbiol 1994; 40: 307-312.

7. Hall E, Parton R, Wardlaw AC. Differences in coughing and other responses to intrabronchial infection with Bordetella pertussis among strains of rats. Infect Immun 1997; 65: $4711-4717$.

8. Hall E, Parton R, Wardlaw AC. Responses to acellular pertussis vaccines and component antigens in a coughing-rat model of pertussis. Vaccine 1998; 16: 1595-1603.

9. Geller BD, Pittman M. Immunoglobulin and histaminesensitivity response of mice to live Bordetella pertussis. Infect Immun 1973; 8: 83-90.

10. Mills KHG, Barnard A, Watkins J, Redhead K. Cell-mediated immunity to Bordetella pertussis: role of Thl cells in bacterial clearance in a murine respiratory infection model. Infect Immun 1993; 61: 399-410.

11. Mayrhofer G, Bazin H, Gowans JL. Nature of cells binding anti-IgE in rats immunized with Nippostrongylus brasiliensis: IgE synthesis in regional lymph nodes and concentration in mucosal mast cells. Eur $J$ Immunol 1976; 6: 537-545.

12. Abraham SN, Malaviya $R$. Mast cells in infection and immunity. Infect Immun 1997; 65: 3501-3508.

13. Torre D, Issi M, Chelazzi G, Fiori GP, Sampietro C. Total serum IgE levels in children with pertussis. Am $J$ Dis Child 1990; 144: 290-291.

14. Wirsing von Konig $\mathbf{C H}$, Finger $\mathrm{H}$. Specific IgE antibodies to Bordetella pertussis antigens during whooping cough. Lancet 1989; 1: 728 .

15. Friedman RL. Pertussis: the disease and new diagnostic methods. Clin Microbiol Rev 1988; 1: 365-376. 